\title{
HOW DO TASK STRUCTURE AND UNCERTAINTY INFLUENCE TASK- INTERLEAVING STRATEGIES DURING DISTRACTED DRIVING?
}

\author{
David Kidd \\ George Mason University \\ Fairfax, Virginia, USA \\ Email: dkidd3@gmu.edu
}

\begin{abstract}
Summary: During distracted driving, people commonly alternate or interleave attention between driving and another task. One factor that influences task interleaving is task structure. Specifically, people tend to switch between tasks at sub-tasks boundaries. Uncertainty about the roadway environment during glances away from the road, however, may play a larger role in shaping task interleaving strategies during distracted driving. The purpose of this study was to examine task interleaving strategies when drivers completed a distracting task of various subtask sizes. Participants entered phone numbers, modified zip codes, or digit strings while performing a lane-keeping task. In general, the time between button presses in the secondary task was significantly greater between sub-tasks than within sub-tasks. However, as sub-tasks became larger drivers switched more frequently within sub-tasks than between sub-tasks. Additionally, participants' did not change their visual sampling strategies as the size of sub-tasks increased. Thus, uncertainty influenced decisions to switch between two interleaved tasks in the driving environment more than sub-task boundaries.
\end{abstract}

\section{INTRODUCTION}

When drivers perform a secondary task while driving, they generally alternate attention and vision between the two tasks in a sequential fashion. In this sense, they are interleaving performance on the two separate tasks. There are a number of ways that drivers could interleave a secondary task with driving. In the case of dialing a phone number, the driver could perform little to no interleaving and dial the entire phone number during a single, extended glance away from the roadway. Drivers, however, are reluctant to neglect the driving task for long periods of time (Klauer et al., 2006) making this type of strategy unlikely. Alternatively, the driver could break up the phone number into smaller portions that could be dialed during a series of glances to and from the roadway. The interleaving strategy the driver chooses influences performance and safety, so it is important to understand the factors underlying task interleaving strategies. Task interleaving strategies are influenced by cognitive constraints and task demand characteristics. For example, people tend to interleave at boundaries between sub-tasks (Payne, Duggan, \& Neth, 2007). Sub-task boundaries provide an opportune time to interleave because cognitive workload is lower at sub-task boundaries (Bailey \& Iqbal, 2008) and it is easier to resume a task at sub-task boundaries (Monk, Boehm-Davis, \& Trafton, 2004). Brumby, Salvucci, and Howes (2009) found that drivers interleaved a distracting dialing task at sub-task boundaries. In their study, drivers dialed a United States (US) phone number (e.g., 012-345-6789) while performing a lane-keeping task. When prioritizing lane-keeping performance, there was a significantly greater amount of time between keystrokes at sub-task boundaries (e.g., following the 2 and the 5) compared to other points in the dialing task. 
The time that visual attention is directed away from the roadway may also affect task interleaving strategies. Senders, Kristofferson, Levison, Dietrich, and Ward’s (1967) uncertainty model suggests that drivers' visual sampling strategies reflect the amount of uncertainty about the roadway environment. Drivers only glance away from the roadway for as long as their uncertainty about the roadway environment remains at an acceptable level. Brumby et al. (2009) found that drivers interleaved at sub-task boundaries, however, a US phone number contains three relatively small sub-tasks that do not require long glances away from the roadway before a sub-task boundary is reached. In a study where drivers dialed a United Kingdom phone number that contained larger sub-tasks (012345-678910), drivers interleaved at points other than the sub-task boundary (Janssen \& Brumby, 2009). Drivers generally dialed in 2 or 3 digit chunks, therefore interleaving at points other than sub-task boundaries, a strategy that would minimize uncertainty about the roadway environment.

Previous research has provided mixed evidence that task interleaving during distracted driving primarily occurs at sub-task boundaries. Support for a sub-task boundary based strategy may have benefited from a distracting task with small sub-tasks (e.g., Brumby et al. 2009). The purpose of this study was to further examine the role sub-task boundaries play in task interleaving during distracted driving using a distraction task with various sized sub-tasks. In this study, drivers entered US phone numbers, modified US zip codes, or digit strings while completing a lane-keeping task. If sub-task boundaries were the primary factor behind task interleaving strategies then switches from the number entry task to the lane-keeping task were expected to be more frequent at sub-task boundaries compared to other locations in the task. Also, the latency between button presses at digit positions representing a sub-task boundary in a number stimulus should be significantly longer compared to the same digit position that is not a sub-task boundary in the other number stimuli. On the other hand, if task interleaving strategies were primarily driven by time and uncertainty about the roadway environment then drivers would switch back to the lane-keeping task at other points in the number entry task and limit the time the roadway view was occluded regardless of the type of number they entered.

\section{METHOD}

Forty-eight (16 men, 32 women) George Mason University undergraduate students were recruited to participate in this study. Participants were between 18 and 40 years old and were 21 years $(S D=4.8)$ old on average. All participants had at least 2 years of driving experience and were fully licensed drivers. All participants reported owning a cell phone and 88 percent reported using their cell phone while driving. Participants received course credit for participating.

\section{Apparatus and Tasks}

This study was conducted using a desktop driving simulator by Realtime Technologies, Inc. (RTI). RTI's SimVista authoring tool was used to create the simulated driving scenario and experimental tasks. The simulated environment was run using RTI's SimCreator software. All visuals were presented on a 20 -inch LCD monitor at 60 frames per second. Participants controlled the simulated vehicle using a Logitech MOMO force feedback racing wheel and pedals. The steering wheel was equipped with two response paddles that were used in the secondary task. A numeric keypad used to enter numbers in the secondary task was mounted to 
the right of the steering wheel. The numeric keypad key layout was inverted to match the key layout of a cellular phone. Driving and secondary task performance were collected at $100 \mathrm{~Hz}$. Participants completed two tasks: a lane-keeping task and a number entry task. In the lanekeeping task participants drove their vehicle along a straight, four-lane roadway (see Figure 1a). The simulated vehicle's speed was fixed at 40 miles per hour (mph). Participants were instructed to keep their vehicle as close to the center of the right lane as possible. The boundaries of the right lane were marked with standard lane lines and construction pylons. The construction pylons were used to discourage participants from deviating out of the lane.

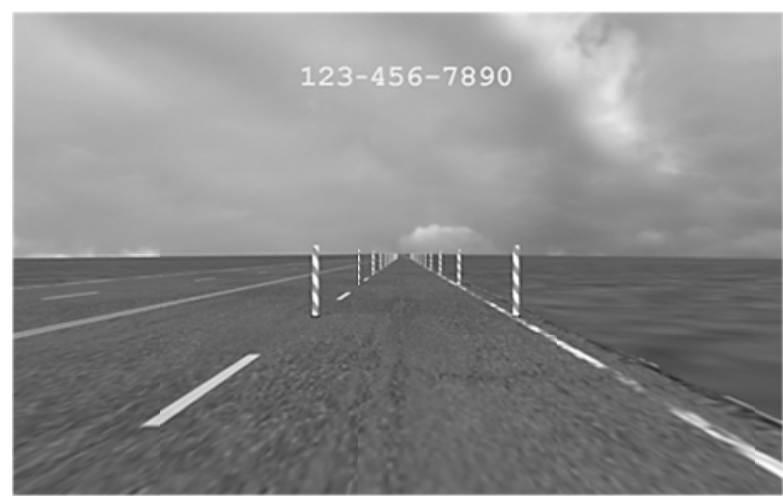

a

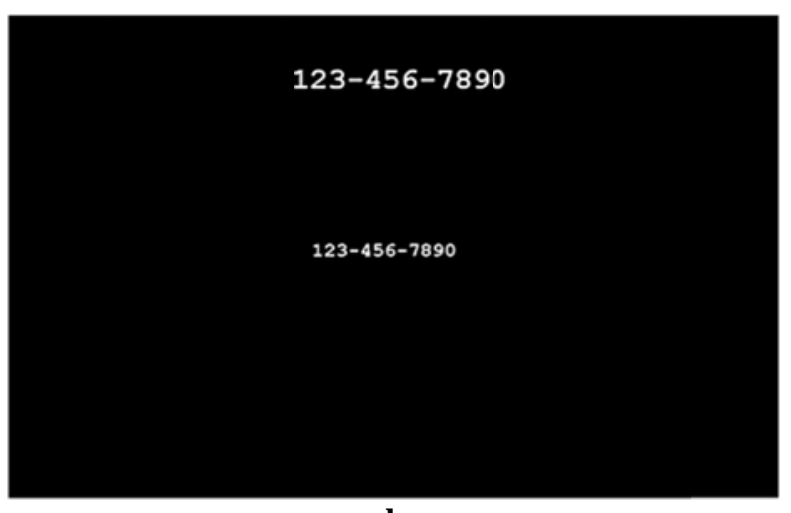

b

Figure 1. (a) Lane-keeping task with a number entry stimulus displayed and (b) occluded roadway scene during number entry

In the number entry task, participants entered a string of numbers using the numeric keypad. Participants were randomly assigned to enter one of three types of number stimuli: (1) a US phone number (XXX-XXX-XXXX), (2) a modified zip code (XXXXX-XXXXX), and (3) a number string (XXXXXXXXXX). Each type of stimulus was 10 digits long, but differed in subtask size and number of sub-task boundaries. The phone number had 2 sub-task boundaries, the zip code had 1 sub-task boundary, and the number string did not have a sub-task boundary. All of the stimuli were randomly generated with the constraints that no two consecutive digits were identical. Also the zip codes and phone numbers did not exist.

Number entry task stimuli were displayed at the top of the LCD monitor. A self-paced occlusion paradigm was used to allow participants to complete the number entry task. Participants could only enter numbers when the roadway scene was occluded. Participants occluded the roadway scene by pulling on one of the two response paddles mounted behind the steering wheel. When the roadway scene was occluded a black screen covered the roadway scene and participants were able to enter numbers (see Figure 1b). Number entry task stimuli were still available during the occlusion period to minimize working memory demand. Participants were instructed to replicate the number entry stimuli using the keypad and then pressed the "Send" button to submit their response. Dashes at the sub-task boundaries were automatically inserted, so participants only had to enter the numbers in each stimulus. 


\section{Procedure}

At the beginning of the study participants completed a demographic questionnaire. Participants were then introduced to the driving simulator and completed a 5-minute familiarization scenario. Next, participants were trained on the number entry task and practiced it by entering 6 number stimuli that were not used in the experiment.

Next, participants completed 5 driving scenarios -2 single-task and 3 dual-task scenarios. Participants first performed the number entry task and the lane-keeping task independent of one another (single-task scenarios). For the number entry single-task scenario, participants' vehicle remained in park and they were instructed to occlude the roadway scene for the duration of the scenario while they entered 12 number stimuli. Participants performed the lane-keeping task for 5 minutes in the lane-keeping only single-task scenario.

After completing the single-task scenarios, participants performed the lane-keeping task and number entry task together. Participants entered 12 number stimuli in each dual-task scenario. Number stimuli were randomly selected without replacement from over 70 possible stimuli for each stimulus type. After entering a number stimulus, participants were given 15-seconds to stabilize their lane position before the next number stimulus appeared. Participants were told that they could use any strategy they wished to interleave the two tasks. They were also instructed to prioritize driving safety and lane-keeping task performance. Each dual-task drive took about 10 minutes. After the dual-task scenarios, participants completed an implicit memory test. Participants listed as many of the number stimuli they entered during the dual-task scenarios as they could remember. After the memory test, participants were debriefed and dismissed.

\section{RESULTS}

Data from the first dual-task scenario were not included because it was participants' first exposure to dual-task performance. Inaccurate trials, trials where the number was entered incorrectly or where a mistake was made during entry, were also removed ( $10 \%$ of all data). The remaining data was aggregated across trials for each participant.

Performing the number entry task while driving significantly increased both lane variability, $F(1$, $46)=13.1, p<.001$, and average deviation from lane center, $F(1,46)=13.1, p<.05$; however, lane-keeping performance did not vary reliably by number entry stimulus or over time during entry. Thus, the analyses below focused on number entry task performance to evaluate participants' task interleaving strategies.

\section{Task Interleaving Strategies}

Two different measures were used to characterize participants' task interleaving strategies: interbutton interval (IBI) and switch location. IBI was the time between two consecutive key presses in the number entry task. IBI is expressed in terms of dual-task cost or dual-task IBI minus single-task IBI for each digit position. Average IBI for all three number entry stimuli at all 10 digit positions is shown in Figure 2. Note that IBI at digit position 1 is the average time it took participants to start entering the number stimulus after the stimulus appeared. 
There were two sub-task boundaries in the phone number stimulus (digit positions 4 and 7) and one in the zip code stimulus (digit position 6). IBI at each of these digit positions across number entry task stimuli was analyzed using a 3 sub-task boundary location (digit positions 4, 6, and 7) x 3 number stimulus (phone, zip code, number string) mixed factorial Analysis of Variance (ANOVA). The ANOVA revealed a significant interaction between sub-task boundary location and number stimulus, $F(4,90)=24.4, p<.001$. IBI at the first sub-task boundary, digit position 4 , was significantly greater in the phone stimulus compared to the string stimulus, $p<.01$. No reliable difference was found between the zip code stimulus and phone stimulus, $p=.10$.

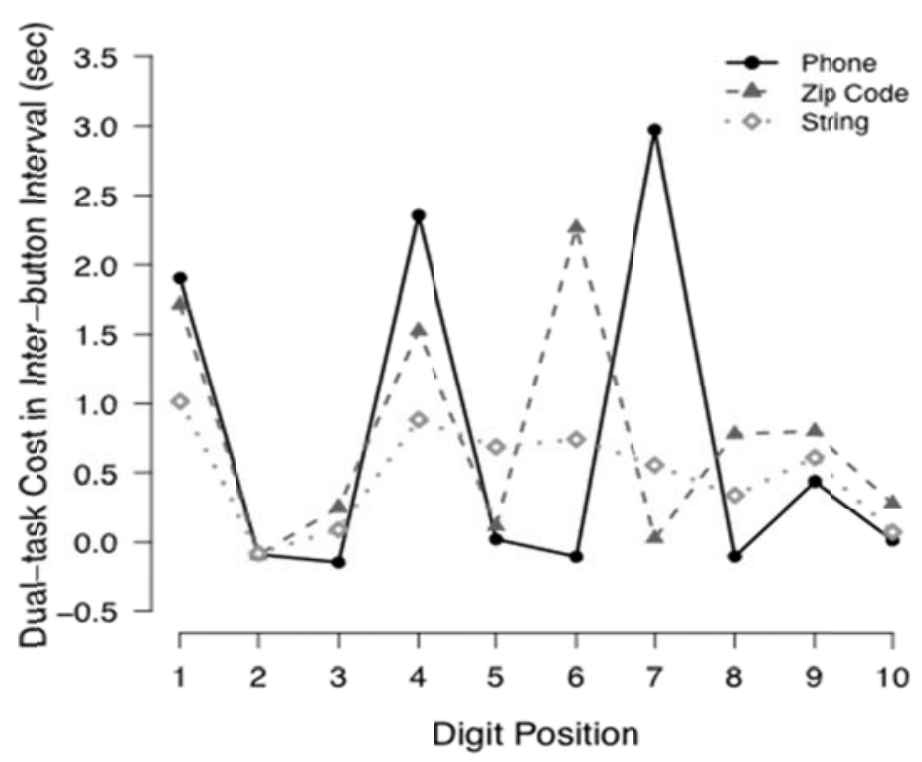

Figure 2. Average dual-task cost of IBI at each digit position for all three number entry stimuli

The sub-task boundary in the zip code stimulus was at digit position 6. As expected, IBI was significantly greater for the zip code stimulus than both the phone number and string stimuli, $p<$ .001 and $p<.01$ respectively. Unexpectedly, average IBI in the string stimulus was significantly slower than the phone stimulus, $p<.01$. Digit position 7 was the second sub-task boundary in the phone number stimulus. Average IBI in the phone stimulus was significantly greater than both the zip code and string stimuli, $p$ 's $<.001$. The difference between average IBI in the zip code and string stimuli at digit position 7 approached significance, $p=.06$. In summary, for the most part average IBI was generally greater in the number stimulus condition with a sub-task boundary compared to the number stimulus conditions without a sub-task boundary at digit positions 4,6 , and 7.

Switches from the number entry task back to the lane-keeping task were also recorded. Switches either occurred at sub-task boundaries (between sub-tasks) or during sub-tasks (within subtasks). The average percent of all switches that occurred between and within sub-tasks was calculated across all trials completed by each individual. The string stimulus was not included in this analysis because it did not have any sub-task boundaries. A 2 number stimulus (Phone, Zip Code) x 2 switch location (Between sub-task, Within sub-task) mixed factorial ANOVA revealed a significant interaction between switch location and number entry stimulus, $F(1,30)=60.9, p<$ .001 . In the phone number condition, participants switched at the sub-task boundaries over $80 \%$ 
of the time (see Table 1). In the zip code condition, however, participants only switched within sub-task boundaries two thirds of the time. This finding suggests that participants did not restrict task interleaving to sub-task boundaries especially when entering the zip code stimuli.

Table 1. Mean percent of total switches by switch location

\begin{tabular}{lcc}
\hline \multirow{2}{*}{ Number Stimulus } & \multicolumn{2}{c}{ Switch Location } \\
\cline { 2 - 3 } & Between & Within \\
\hline Phone & $81.5(19.6)$ & $18.5(20.0)$ \\
Zip Code & $33.4(14.9)$ & $66.6(14.9)$ \\
\hline \multicolumn{2}{c}{ Note: standard deviations in parentheses }
\end{tabular}

\section{Visual Sampling Strategies}

One benefit of using a self-paced occlusion paradigm is that it allowed for precise measurement of participants' visual sampling strategy. Occlusion periods occurred when participants occluded the roadway view to perform the number entry task. The duration of each occlusion period was recorded and averaged across trials for each participant. All participants occluded the roadway at least 3 times to enter the number entry stimulus. The average duration of the first 3 occlusion periods was compared across number entry stimuli using a 3 occlusion period (first, second, third) $x 3$ number stimulus (phone, zip code, string) mixed factorial ANOVA. The duration of each occlusion period decreased significantly over time, $F(2,90)=7.1, p<.01$. The duration of the first occlusion period $(M=1.13 \mathrm{sec}, S D=0.1)$ was significantly longer than the second $(M=$ $1.02 \mathrm{sec}, S D=0.1)$ and third occlusion periods $(M=1.00 \mathrm{sec}, S D=0.1)$. The duration of the first three occlusion periods was not reliably different across number entry task stimuli. This suggests that participants’ visual sampling strategies did not vary across number stimuli.

\section{DISCUSSION}

The purpose of this study was to determine if task interleaving during distracted driving primarily took place at sub-task boundaries. As found in previous research (Brumby et al., 2009), IBI was significantly greater at digit positions that followed a sub-task boundary in a number stimulus compared to the same digit position that did not follow a sub-task boundary in the other number stimuli. A visual inspection of Figure 2, however, clearly shows that IBI increased at digit positions other than sub-task boundaries. When examining the frequency of switches at each digit position, drivers switched within a sub-task $19 \%$ of the time when entering phone numbers and $67 \%$ of the time when entering zip codes. Thus, drivers frequently switched within sub-tasks especially when entering larger sub-tasks such those in the zip code stimuli. This finding replicates previous research (Janssen \& Brumby, 2009) and suggests that drivers did not restrict task interleaving to sub-task boundaries.

Participants' visual sampling patterns provided additional evidence that task interleaving was not primarily influenced by sub-task boundaries. Sub-tasks in each of the number entry stimuli varied in size. If participants interleaved at sub-task boundaries then the average duration of occlusion periods should have been greater in stimuli with larger sub-tasks (i.e., zip code and 
string). The average duration of participants' first three occlusion periods was not significantly different across number entry stimuli. On average, participants only occluded the roadway between 1 and 1.2 seconds, durations that have been found in previous visual sampling research (Tsimhoni \& Green, 2001). This suggests that participants were using a similar visual sampling strategy for each number entry stimulus regardless of sub-task size. Limiting the duration of occlusion periods allowed participants to reduce decrements in lane-keeping performance during entry and minimize uncertainty about the roadway (Senders et al., 1967).

It is important to note several limitations in this study. First, task interleaving behavior was explored in a simplistic driving environment. Lane-keeping is commonly used in research as a proxy for actual driving, but it certainly does not incorporate all of the complexities and inherent risks of actual driving. As such, the task interleaving strategies observed in this study may not fully account for how drivers switch between tasks in actual driving. Second, whereas the selfpaced occlusion paradigm allowed visual sampling strategies to be measured, it is not a suitable substitute for eye tracking. The occlusion method, however, is commonly used to measure and estimate visual demand (e.g., Baumann, Keinath, Krems, \& Bengler, 2004) and the average occlusion period durations in this study were similar to glance durations observed in previous research (Tsimhoni \& Green, 2001).

In summary, the current findings suggested that drivers did not constrain task interleaving to subtask boundaries in the number entry task. Drivers switched from the number entry task to the lane-keeping task in the middle of sub-tasks and used a similar visual sampling strategy when completing sub-tasks of different sizes. Thus, contrary to previous research (Brumby et al., 2009), task interleaving was influenced by the time that drivers were willing to keep their visual attention away from the roadway and not sub-task boundaries in the number entry task.

\section{REFERENCES}

Bailey, B. P., \& Iqbal, S. T. (2008). Understanding changes in mental workload during execution of goal-directed tasks and its application for interruption management. ACM Trans. Comput.Hum. Interact., 14 (4), 1-28.

Baumann, M., Keinath, A., Krems, J. F., \& Bengler, K. (2004). Evaluation of in-vehicle HMI using occlusion techniques: experimental results and practical implications. Applied Ergonomics, 35(3), 197-205.

Brumby, D. P., Salvucci, D. D., \& Howes, A. (2009). Focus on driving: how cognitive constraints shape the adaptation of strategy when dialing while driving. In Proceedings of the 27th international conference on human factors in computing systems (pp. 1629-1638). New York, NY, USA: ACM.

Klauer, S. G., Dingus, T. A., Neale, V. L., Sudweeks, J. D., \& Ramsey, D. J. (2006, April). The impact of driver inattention on near-crash/crash risk: An analysis using the 100-car naturalistic driving study data (DOT HS 810 594). Washington, D.C.: NHTSA.

Monk, C. A., Boehm-Davis, D. A., \& Trafton, J. G. (2004). Recovering from interruptions: Implications for driver distraction research. Human Factors, 46 (4), 650-663. 
Payne, S. J., Duggan, G. B., \& Neth, H. (2007). Discretionary task interleaving: Heuristics for time allocation in cognitive foraging. Journal of Experimental Psychology: General, 136(3), 370-388.

Senders, J., Kristofferson, A., Levison, W., Dietrich, C. W., \& Ward, J. L. (1967). The attentional demand of automobile driving. Highway Research Record, 195, 15-33.

Tsimhoni, O. \& Green, P. (2001). Visual demand of driving and the execution of Display Intensive In-Vehicle tasks. Human Factors and Ergonomics Society Annual Meeting Proceedings , 45, 1586-1590. 